\title{
Average minimum transmit power to achieve SINR targets: performance comparison of various user selection algorithms
}

\author{
Umer Salim $^{1 *}$ and Dirk Slock ${ }^{2}$
}

\begin{abstract}
In multi-user communication from one base station (BS) to multiple users, the problem of minimizing the transmit power to achieve some target-guaranteed performance (rates) at users has been well investigated in the literature. Similarly, various user selection algorithms have been proposed and analyzed when the BS has to transmit to a subset of the users in the system, mostly for the objective of sum rate maximization. We study the joint problem of minimizing the transmit power at the BS to achieve specific signal-to-interference-and-noise ratio (SINR) targets at users in conjunction with user scheduling. The general analytical results for the average transmit power required to meet guaranteed performance at the users' side are difficult to obtain even without user selection due to joint optimization required over beamforming vectors and power allocation scalars. We study the transmit power minimization problem employing non-linear dirty paper coding (DPC) technique and with various user selection algorithms, namely semi-orthogonal user selection (SUS), norm-based user selection (NUS), and angle-based user selection (AUS). Starting from the derivation of a transmit power upper bound (that becomes tight for large SINR targets), the average minimum transmit power is derived for NUS and SUS, for any number of users. For the special case when only two users are selected, we further derive a similar expression for AUS and a power lower bound, which may serve to benchmark the performance of any selection scheme. Simulation results performed under various settings indicate that SUS is by far the better user selection criterion.
\end{abstract}

\section{Introduction}

\section{A. Motivation}

In multi-antenna downlink (DL) systems, the characterization of the capacity (rate) regions and the maximization of the sum rate have been among the most widely studied subjects. The capacity region of DL singleantenna systems was first studied by Cover in [1]. After the discovery of spatial multiple antenna gains for single-user (SU) systems in [2,3], the focus of research shifted to multiple antenna multi-user (MU) systems. Conditioned upon the availability of perfect channel state information (CSI), the capacity region of multiantenna DL channel is known [4-7], and hence the optimal (dirty paper coding (DPC), first proposed in [8] was shown to be the optimal strategy in [4]) and a wide variety of suboptimal (but less complicated) transmission

\footnotetext{
* Correspondence: umer.salim@intel.com

${ }^{1}$ Intel Mobile Communications, France

Full list of author information is available at the end of the article
}

strategies have been proposed and analyzed. In many practical wireless systems, maximizing the throughput may not be the primary objective. A very important design objective for multi-antenna MU systems is to achieve a particular link quality over all links with minimum transmission power, which is equivalent to achieving certain signal-to-interference-and-noise ratios (SINR) or data rates over corresponding links. This problem, in some sense, is the dual problem of the sum rate maximization under a fixed power constraint. Certainly, from an operator's perspective, the minimization of average transmit power to achieve these SINR targets is of prime importance.

Combined MU transmission with user scheduling has been widely analyzed in the sum rate maximization perspective (see [9-12] and the references therein), but very rarely for the objective of the transmit power minimization. Very pertinent questions in this area include how does the minimum average transmit power decay with the number of users or the number of BS transmit 
antennas. Similarly, the optimal user selection scheme for transmit power minimization has never been investigated. In the context of the sum rate maximization, the semi-orthogonal user selection (SUS) has been shown to behave asymptotically optimal [11] and is widely believed to be the best greedy user selection strategy $[9,10,12]$, but no such study has been conducted for the transmit power optimization problem with hard SINR targets, and no analytical results for average transmit power are known. Hence, the characterization of the average minimum transmit power for various user selection mechanisms and relative performance comparisons are very relevant research objectives.

\section{B. The state of the art}

The problem of minimizing the DL transmit power required to meet users' SINR constraints by joint optimization of transmit beamforming $(\mathrm{BF})$ vectors and power allocation scalars was first solved in [13] and was later treated in $[14,15]$ with feasibility issues. These solutions are based upon the duality of uplink (UL) and DL channels. Exploiting this UL-DL duality, iterative algorithms were proposed to find the optimal BF vectors and the optimal power assignments to the users, and the convergence of these algorithms was shown to the optimal solution. For MU channels (either UL or DL) with Gaussian signalling, [15] showed that the problem of minimizing the transmit power to achieve specific SINR targets bears a relatively simple solution due to the added structure that may be exploited by successive interference cancellation (SIC) in the UL and by DPCbased encoding for known interference in the DL channels, and the results were presented in [15-17]. The optimal BF strategy turns out to be the minimummean-square-error (MMSE) solution, where each user will see no interference from the already encoded users, due to DPC-based encoding and each BF treats the interference of unencoded users as extra noise, and power allocation for each user is done to raise its SINR level to the target SINR. Actually, the DL problem is solved by first solving the dual UL problem, due to its relatively simple structure.

There is a line of work by Karipidis et al., where they studied QoS and Max-Min fair transmit BF for multiple multicast groups $[18,19]$, showing the original problem to be NP-hard and proposing solutions for relaxed versions. This work essentially differs from our work as we stick to the DL channel, but investigate the performance of user selection algorithms with the criterion of average transmit power fulfilling hard SINR targets.

The performance of different user selection algorithms for transmit power minimization was studied in [20] ([21] is the journal version). The Gaussian MU systems were analyzed without exploiting the extra system structure through SIC or DPC, when SINR targets are large. They obtained analytical expressions for the average minimum transmit power required for guaranteed rates with norm-based user selection (NUS) and anglebased user selection (AUS) in the limiting case, when only two users are selected. For the same scenario of two selected users, the expressions for average minimum transmit power were derived for NUS, AUS, and SUS employing SIC (in UL) or DPC (in DL) in [22].

\section{Contribution}

We study the problem of average transmit power minimization to meet users' SINR constraints in conjunction with user scheduling. In this MU system with Gaussian signalling, we make use of DPC-based encoding in the DL channel. As the channel information is already required at the $\mathrm{BS}$ for $\mathrm{BF}$ and power assignments, this additional processing does not require any extra information. This problem formulation gives twofold advantage over [20]: first, no iterations are required to compute the optimal BF vectors and power allocation scalars and second, less average power is required at the transmitter to satisfy the same SINR constraints. On the negative side, DPC encoding is computationally cumbersome [11], and practical DPC code design is still an active area of research (see [23,24] and references therein). We first derive an upper bound of the transmit power required to satisfy users' SINR targets that is shown to be tight for large SINR targets. Based upon this bound, the average minimum transmit power expressions are derived for any number of users selected through SUS, NUS, or random user selection (RUS). For the case of two active users, we derive similar analytical expression with AUS. A lower bound of the average minimum transmit power is also derived for the twouser case, which may serve to benchmark any user selection mechanism. Detailed performance comparison is provided for these user selection schemes with derived results and Monte Carlo simulations. It turns out that NUS and AUS are strictly suboptimal when compared with SUS.

For the objective of the minimization of transmit power to achieve hard SINR targets, the optimal DPC encoding order is an open problem, although numerical simulations indicate that a good choice of encoding order is such that the weaker user gets decoded with least interference [15]. The optimization of encoding order is out of the scope of this paper, and we stick to the above-defined encoding strategy.

Some may argue that in a scenario with SINR requirements for all users, all these users need to be scheduled. In that case, the problem of user scheduling becomes in fact a problem of user permutation to determine which users are going to be scheduled together within a certain 
(time-frequency in OFDMA) resource block, with all users being scheduled on one or another resource block. The optimization of such a user assignment can only be attained by a computationally exhaustive search, which calls for greedy suboptimal approaches. In one such greedy approach, the user selection gets performed per resource block, sequentially treating the various resource blocks. This means that for a given resource block, the user selection gets performed from a pool of users, the size of which is decreasing as we progress through the list of resource blocks. The selection process for each resource block is then of the form considered here. But as the size $K$ of the pool of users to choose from is varying, we analyze the user selection process for varying $K$.

\section{Organization}

This contribution is organized as follows. Section 2 describes the system model. Section 3 gives a brief overview of the problem of transmit power minimization without user selection. In Section 4, certain user selection algorithms are reviewed, for which later we analyze the performance. The main results of the paper, the analytical expressions for the average minimum transmit power for different user selection schemes, are presented in Section 5. The proof details have been relegated to appendices to keep the subject material simple and clear. The performances of these user selection algorithms are compared in Section 6 followed by the concluding remarks in Section 7.

NOTATION: Lowercase letters represent scalars, boldface lowercase letters represent vectors, and boldface uppercase letters denote matrices. $\mathbf{A}^{\dagger}$ denotes the Hermitian transpose of matrix A. The identity matrix of $n$ dimensions is denoted by $\mathbf{I}_{\mathrm{n}}$. $\mathbb{E}$ denotes statistical expectation. For a random variable $x$ having the cumulative distribution function (CDF) of $F$, its expected value is denoted as $\mathbb{E}_{F}[x]$.

\section{System model}

The system, we consider, consists of a BS having $M$ transmit antennas and $K$ single-antenna user terminals. In the DL, the signal received by $k$ th user can be expressed as

$$
y_{k}=\mathbf{h}_{\mathbf{k}}^{\dagger} \mathbf{x}+z_{k}, \quad k=1,2, \ldots, K
$$

where $\mathbf{h}_{1}^{\dagger}, \mathbf{h}_{2}^{\dagger}, \ldots, \mathbf{h}_{\mathbf{K}}^{\dagger}$ are the channel vectors of users 1 through user $K$ with $\mathbf{h}_{\mathbf{k}} \in \mathbb{C}^{M \times 1}$ and $\mathbf{x} \in \mathbb{C}^{M \times 1}$ denotes the signal transmitted by the BS. The terms $z_{1}$, $z_{2}, \ldots, z_{K}$ represent independent zero-mean complex Gaussian additive noise terms. The system parameters have been normalized such that every user suffers from the unit variance noise. We denote the concatenation of the channels by $\mathbf{H}_{\mathrm{F}}^{\dagger}=\left[\mathbf{h}_{1}, \mathbf{h}_{2}, \ldots, \mathbf{h}_{\mathbf{K}}\right]$, so $\mathbf{H}_{\mathbf{F}}$ is $K \times M$ forward channel matrix with $k$ th row equal to the channel of $k$ th user $\left(\mathbf{h}_{\mathbf{k}}^{\dagger}\right)$. The channel is assumed to be block fading, staying constant for its coherence length and then changing to an independent state. The entries of the forward channel matrix $\mathbf{H}_{\mathbf{F}}$ are i.i.d. complex Gaussian with zero mean and unit variance. We make the simplifying assumption of the presence of perfect CSI at the transmitter (CSIT), so as to focus completely on the performance of different user selection algorithms.

The SINR constraints of the users are denoted by $\gamma_{1}$, $\gamma_{2}, \ldots, \gamma_{K}$. As SINR is a direct measure of the successful signal decoding capability at a receiver (user), these constraints can be easily translated to rate constraints. If $K_{s}$ out of $K$ users (implying $K_{s}<K$ ) are selected for transmission during each coherence interval, the channel input $\times$ can be written as $\mathbf{x}=\overline{\mathbf{V}} \mathbf{P}^{1 / 2} \mathbf{u}$, where $\overline{\mathbf{V}} \in \mathbb{C}^{M \times K_{s}}$ denotes the beamforming matrix with normalized columns, $\mathbf{P}$ is $K_{s} \times K_{s}$ diagonal power allocation matrix with positive real entries, and $\mathbf{u} \in \mathbb{C}^{K_{s} \times 1}$ is the vector of zero-mean unit-variance Gaussian information symbols. Hence, $\mathbb{E}[\operatorname{Tr}(\mathbf{P})]$ is the average transmit power that can be minimized by optimizing over the beamforming matrix $\overline{\mathbf{V}}$, and the power allocation matrix $\mathbf{P}$ to achieve the SINR targets. We select this average minimum transmit power as the performance metric and study the performance of various user selection algorithms when users' SINR targets need to be satisfied.

\section{Overview of transmit power minimization problem}

The signal received by $k$ th user can be written as

$$
\begin{aligned}
y_{k} & =\mathbf{h}_{\mathbf{k}}^{\dagger} \overline{\mathbf{V}} \mathbf{P}^{1 / 2} \mathbf{u}+z_{k}, \quad k=1,2, \ldots, K_{s} \\
& =\sqrt{p_{k}} \mathbf{h}_{\mathbf{k}}^{\dagger} \overline{\mathbf{v}}_{\mathbf{k}} u_{k}+\sum_{\substack{j=1 \\
j \neq k}}^{K_{s}} \sqrt{p_{j}} \mathbf{h}_{\mathbf{k}}^{\dagger} \overline{\mathbf{v}}_{\mathbf{j}} u_{j}+z_{k},
\end{aligned}
$$

where $p_{k}$ represents the power allocated to the stream of $k$ th user. The second term in the expression represents the interference contribution at $k$ th user, due to the beams meant for other selected users. Based upon this received signal, the SINR of $k$ th user can be written as

$$
\operatorname{SINR}_{\mathbf{k}}=\frac{p_{k}\left|\mathbf{h}_{\mathbf{k}}^{\dagger} \overline{\mathbf{v}}_{\mathbf{k}}\right|^{2}}{\sum_{\substack{j=1 \\ j \neq k}}^{K_{s}} p_{j}\left|\mathbf{h}_{\mathbf{k}}^{\dagger} \overline{\mathbf{v}}_{\mathbf{j}}\right|^{2}+1} .
$$

Implicit in this SINR expression is the fact that the users are equipped with simple receivers that do not try to decode the signal of other users, and hence the interference present in the received signal is treated as 
noise. Such receivers are commonly known in the literature as SU receivers $[25,26]$. Without user selection, the problem of optimization of beamforming vectors and power allocation was solved in $[14,15]$ using the UL-DL duality for a fixed encoding/decoding order (see Section 4.3 and 5.2 in [15] for details). They gave iterative algorithms to obtain the optimal beamforming vectors and the optimal power allocation for each user. The optimal beamforming vectors corresponding to a particular (suboptimal) power allocation are obtained, then power allocations are updated corresponding to these beamforming vectors. This process is repeated till both converge to their optimal values. Unfortunately, general closed-form expressions for the transmit power required to achieve SINR targets do not exist, due to intricate inter-dependence of beamforming vectors and power allocations, as is evident from Equation (3).

For MU systems with Gaussian signalling, if DPCbased successive encoding is employed at the transmitter from $K_{s}$ to 1 , then $k$ th user will only receive the interference of those users, which are encoded after its own data encoding. The signal of already encoded users falls in the paradigm of known interference and can be treated without any power penalty $[4,8]$. Hence, the effective signal received at $k$ th user will be

$$
y_{k}=\sqrt{p_{k}} \mathbf{h}_{\mathbf{k}}^{\dagger} \overline{\mathbf{v}}_{\mathbf{k}} u_{k}+\sum_{j=1}^{k-1} \sqrt{p_{j}} \mathbf{h}_{\mathbf{k}}^{\dagger} \overline{\mathbf{v}}_{\mathbf{j}} u_{j}+z_{k} .
$$

Based upon this received signal, the SINR of $k$ th user can be written as

$$
\operatorname{SINR}_{\mathbf{k}}=\frac{p_{k}\left|\mathbf{h}_{\mathbf{k}}^{\dagger} \overline{\mathbf{v}}_{\mathbf{k}}\right|^{2}}{\sum_{j=1}^{k-1} p_{j}\left|\mathbf{h}_{\mathbf{k}}^{\dagger} \overline{\mathbf{v}}_{\mathbf{j}}\right|^{2}+1} .
$$

For this MU system, the optimal BF vectors and power assignments can be computed using back substitution without any iteration. Although iterations are not required in this scenario, yet beamforming vector and power allocation of one user depend upon the BF vectors and power assignments of already treated users. For the same noise variance at each user, the minimum transmit power required for a particular channel instance is given by the following expression $[15,17]$ :

$$
p_{\mathrm{tx}}\left(\mathbf{h}_{1}, \mathbf{h}_{2}, \ldots, \mathbf{h}_{\mathrm{K}_{\mathrm{s}}}\right)=\sum_{i=1}^{K_{\mathrm{s}}} \frac{\gamma_{i}}{\mathbf{h}_{\mathrm{i}}^{\dagger} \mathbf{Z}_{\mathbf{i}}^{-1} \mathbf{h}_{\mathbf{i}}}
$$

where the columns of $\mathbf{Z}_{\mathbf{i}}$ make a subspace gathering the contributions from the channels of those users, which will produce interference for $i$ th user and is given by the following expression

$$
\mathbf{Z}_{\mathbf{i}}=\mathbf{I}_{\mathbf{M}}+\sum_{j=1}^{i-1} p_{j} \mathbf{h}_{\mathbf{j}} \mathbf{h}_{\mathbf{j}}^{\dagger}
$$

\section{Review of user selection algorithms}

There is a plethora of user selection algorithms in the literature, and hence a comprehensive review is out of the scope of this paper. In this section, we briefly give the overview of three most famous user selection algorithms for which we later study the problem of transmit power minimization and derive the corresponding average power expressions.

\section{A. Greedy semi-orthogonal user selection (SUS)}

The greedy SUS algorithm is outlined as follows:

(1) Let $S$ be an empty space and label all channel vectors unprocessed. From $i=1$ to $K_{s}$

(2) Project all unprocessed channels onto the null space of $\mathcal{S}$

(3) Select the user, $\pi^{S}(i)$, with largest 2-norm projection and label it processed.

(4) Set $S$ to be the span of all processed channel vectors.

This scheduling algorithm was first proposed in [9] and was used in conjunction with DPC, similar to our setting. Later, it was analyzed with linear zero-forcing in [10], and also appears in $[11,12]$ with an additional userremoval step. All these references have shown that it performs very well for sum rate maximization, but it requires complete CSI of all the users.

\section{B. Norm-based user selection (NUS)}

This simplified scheme does not require full CSI, and the users are selected based only upon their channel norms, one scalar value per user. Hence, $K$ users are sorted in descending order of their channel norm values, and the first $K_{s}$ (strongest) users are selected for transmission in each scheduling interval. Thus, NUS index, $\pi^{N}(i)$, corresponds to $i$ th user in the sorted list. This user selection has been studied in [27-29] is an extension of single-user selection from [30] to MU scenarios and is reminiscent of selection diversity based processing [31].

\section{Angle-based user selection (AUS)}

The simplified AUS algorithm is outlined as follows:

(1) The first selected user $\pi^{A}(1)$ is the user with the largest norm. The space $\mathcal{A}$ contains $\pi^{A}(1)$.

(2) Normalize all other channel vectors by their norms and label them unprocessed. From $i=2$ to $K_{s}$ 
(3) Project all unprocessed channels onto the null space of $\mathcal{A}$.

(4) Select the user, $\pi^{A}(i)$, with largest 2-norm projection and label it processed.

(5) Set $\mathcal{A}$ to be the span of all processed channel vectors.

The second normalization step, after the selection of first strongest user, makes the channel-norm effect go away completely, and the selection criterion becomes the mutual orthogonality of users' channel vectors. AUS has been analyzed in $[21,32]$.

\section{Random user selection (RUS)}

The RUS selects the active users independent of their channel realizations. The active users can be selected following a round-robin algorithm for fairness in terms of being in the active pool or based upon users' subscription conditions (the users paying more rates to service providers could be given some kind of priority over other users). RUS may serve more the purpose of judging the performance of other selection schemes than itself being used as a selection scheme.

\section{Transmit power with user selection: main results}

In this section, we give the main results of this paper. We start with the following lemma, which gives an upper bound of the minimum transmit power, given in Equation (6).

Lemma 1 (Minimum Transmit Power to achieve SINR Targets): The minimum transmit power to achieve SINR targets $\gamma_{1}, \gamma_{2}, \ldots, \gamma_{K_{s}}$ for $K_{s}$ users is given by the following expression:

$$
p_{\mathrm{tx}}\left(\mathbf{h}_{1}, \mathbf{h}_{2}, \ldots, \mathbf{h}_{\mathbf{K}_{\mathrm{s}}}\right) \leq \sum_{i=1}^{K_{s}} \frac{\gamma_{i}}{\left\|\mathbf{h}_{\mathbf{i}}\right\|^{2} \sin ^{2} \theta_{(i-1)}}
$$

where $\theta_{(i-1)}$ is the angle which $\mathbf{h}_{\mathbf{i}}$ subtends with the $(i-1)$ dimensional subspace spanned by $\mathbf{h}_{\mathbf{1}}, \mathbf{h}_{\mathbf{2}}, \ldots, \mathbf{h}_{\mathbf{i}-1}$ for $i>1$ and $\theta_{0}=\frac{\pi}{2}$, and this inequality becomes tight with large SINR targets.

Proof: The proof details for this lemma appear in Appendix A.

This lemma about the required transmit power to achieve SINR targets bears a very nice intuitive explanation. It says that the effective channel strength of each user is the energy in the projection of this user's channel when it is projected on the null space of its interference subspace, the subspace spanned by the channels of those users who create interference for this user as a function of encoding order. In our setting, where encoding order is $K_{s}$ to 1 , the interference subspace for user $i$ is the subspace spanned by the channels of users $1,2, \ldots, i-1$.
Then each user is allocated the minimal power corresponding to its effective channel energy such that it achieves its SINR target. The sum of these powers gives the minimum transmit power required to achieve SINR targets at $K_{s}$ active users.

Another very important remark about this lemma is that the term appearing in the denominator $\left\|\mathbf{h}_{\mathbf{i}}\right\|^{2} \sin ^{2}$ $\theta_{(i-1)}$ is the orthogonalized squared norm-the metric of greedy SUS algorithm proposed in 4-A. The greedy selection algorithm selects one user in each iteration having the maximum orthogonal norm, thus requiring the minimum power to get its SINR target satisfied. This makes the SUS algorithm the greedy algorithm for power minimization objective.

Now we present further results, the analytical expressions for the average minimum transmit power required to achieve SINR targets at users when these users are selected obeying different user selection algorithms as detailed in Section 4. For these selected users, the optimal beam-forming vectors and the power assignments are computed as outlined in Section 3. We restrict the users to have the same SINR targets $\gamma$. Otherwise, the users with smaller SINR targets become relatively better candidates compared to those with higher targets for the objective of transmit power minimization.

Theorem 1 (Average Minimum Transmit Power for NUS): Consider a DL system having a BS equipped with $M$ transmit antennas and $K$ single-antenna users, each having an SINR constraint of $\gamma$, and $K_{s}$ active users are selected for simultaneous transmission from the pool of $K$ users in each coherence block. If the active users are chosen through NUS, then the average minimum transmit power, denoted as $p_{\mathrm{N}}\left(K_{s}, K\right)$, is bounded as:

$$
p_{\mathrm{N}}\left(K_{s}, K\right) \leq \gamma \sum_{i=1}^{K_{s}}\left(\mathbb{E}_{F_{||||_{\mid} \mid}\left(M, K_{s}+1-i, K ; x\right)}\left[\frac{1}{x}\right] \mathbb{E}_{F_{\text {sin }^{2} \theta_{(i-1)}}(M ; x)}\left[\frac{1}{x}\right]\right)
$$

where $F_{\|\mathbf{h}\|^{2}}(M, r, K ; x)$ denotes the cumulative distribution function (CDF) of $r$ th order statistic of squared norm among $K$-independent $M$-dimensional complex Gaussian vectors, and $F_{\sin ^{2} \theta_{j}}(M ; x)$ denotes the CDF of $\sin ^{2} \theta_{j}$ where $\theta_{j}$ is the angle that an $M$-dimensional vector subtends with an independent $j$-dimensional subspace. Further, this bound becomes tight for large SINR targets.

Proof: The proof details for Theorem 1 and its associated Corollaries 2 and 3 appear in Appendix C.

Corollary 2 (NUS for 2 Users): When $K_{s}=2$ active users are selected through NUS in each coherence block, the average minimum transmit power to achieve SINR target $\gamma$ is bounded as:

$$
p_{\mathrm{N}}(2, K) \leq \gamma\left(K \alpha_{M, K-1}-\left(K-2-\frac{1}{M-2}\right) \alpha_{\mathrm{M}, K}\right) .
$$


where $\alpha_{\mathrm{M}, \mathrm{K}}$ is a constant solely governed by $M$ and $K$ and is defined to be

$$
\alpha_{\mathrm{M}, \mathrm{K}} \triangleq \int_{0}^{\infty} K \frac{e^{-x} x^{M-2}}{\Gamma(M)}[G(M, x)]^{K-1} d x
$$

where $\Gamma(M)$ and $G(M, x)$ denote the Gamma function and the regularized Gamma function [33], respectively.

Corollary 3 (NUS for 4 Users): When $K_{s}=4$ active users are selected through NUS in each coherence block, the average minimum transmit power to achieve SINR target $\gamma$ is given by:

$$
\begin{aligned}
& p_{\mathrm{N}}(4, K) \leq \gamma\left[\frac{M-1}{M-4} \alpha_{M, K}+\left\{K \alpha_{\mathrm{M}, K-1}-(K-1) \alpha_{\mathrm{M}, K}\right\} \frac{M-1}{M-3}+\right. \\
& \left\{\frac{K(K-1)}{2} \alpha_{M, K-2}-K(K-2) \alpha_{M, K-1}+\frac{(K-1)(K-2)}{2} \alpha_{M, K}\right\} \frac{M-1}{M-2}+ \\
& \left.\left\{\frac{K(K-1)(K-2)}{6} \alpha_{M, K-3}-\frac{K(K-1)(K-3)}{2} \alpha_{\mathrm{M}, K-2}+\frac{K(K-2)(K-3)}{2} \alpha_{M, K-1}-\frac{(K-1)(K-2)(K-3)}{6} \alpha_{M, K}\right\}\right] .
\end{aligned}
$$

Theorem 4 (Average Minimum Transmit Power for $S U S)$ : For an $M$-transmit antenna BS and $K$ singleantenna users, if $K_{s}$ active users are selected through SUS for simultaneous transmission, each having an SINR constraint of $\gamma$, the average minimum transmit power, denoted by $p_{S}\left(K_{s}, K\right)$, is bounded as:

$$
p_{\mathrm{S}}\left(K_{s}, K\right) \leq \gamma \sum_{i=1}^{K_{s}}\left(\mathbb{E}_{F_{\|\left.\mathbf{h}\right|^{2}}(M+1-i, i, K ; x)}\left[\frac{1}{x}\right]\right) .
$$

Proof: The proof details for Theorem 4 and Corollaries 5 and 6 appear in Appendix D.

Corollary 5 (SUS for 2 Users): When $K_{s}=2$ active users are selected through SUS in each block, the upper bound of average minimum transmit power to achieve SINR target is given by:

$$
p_{\mathrm{S}}(2, K) \leq \gamma\left(\alpha_{\mathrm{M}, \mathrm{K}}+K \alpha_{\mathrm{M}-1, \mathrm{~K}-1}-(K-1) \alpha_{\mathrm{M}-1, \mathrm{~K}}\right) .
$$

Corollary 6 (SUS for 4 Users): When $K_{s}=4$ active users are selected through SUS in each coherence block, the average minimum transmit power to achieve the SINR targets is:

$$
\begin{aligned}
& p_{\mathrm{s}}(4, K) \leq \gamma\left[\alpha_{\mathrm{M}, K}+K \alpha_{\mathrm{M}-1, K-1}-(K-1) \alpha_{M-1, K}+\frac{K(K-1)}{2} \alpha_{M-2, K-2}-K(K-2) \alpha_{\mathrm{M}-2, K-1}\right. \\
& +\frac{(K-1)(K-2)}{2} \alpha_{M-2, K}+\frac{K(K-1)(K-2)}{6} \alpha_{M-3, K-3}-\frac{K(K-1)(K-3)}{2} \alpha_{M-3, K-2}+\frac{K(K-2)(K-3)}{2} \alpha_{M-3, K-1} \\
& \left.-\frac{(K-1)(K-2)(K-3)}{6} \alpha_{M-3, K}\right] .
\end{aligned}
$$

Theorem 7 (Average Minimum Transmit Power for RUS): For an $M$-antenna BS having $K$ single- antenna users in the pool, when $K_{s}$ active users are selected randomly for simultaneous transmission, the average minimum transmit power required, denoted by $p_{\mathrm{R}}\left(K_{s}, K\right)$, so that each of $K_{s}$ users achieves its SINR target $\gamma$ is given by:

$$
p_{\mathrm{R}}\left(K_{s}, K\right)=p_{\mathrm{N}}\left(K_{s}, K_{s}\right)
$$

Proof: As RUS is independent of channel realizations, the selected users show the same statistics as if there is no user selection. This behavior can be obtained when the pool of available users to NUS is of size $K_{s}$, hence there is no user selection and we get the average power required for RUS.

Theorem 8 (Average Minimum Transmit Power for $A U S)$ : Consider a DL system having a BS equipped with $M$-transmit antennas and $K$ single-antenna users, each having an SINR constraint of $\gamma$, and $K_{s}=2$ users are selected for simultaneous transmission in each coherence block. If the user selection is done through AUS, the average minimum transmit power is bounded as:

$$
p_{\mathrm{A}}(2, K) \leq \gamma\left(\frac{1}{K-1}\left(\frac{K}{M-1}-\alpha_{\mathrm{M}, \mathrm{K}}\right)+\frac{(M-1)(K-1) \alpha_{\mathrm{M}, \mathrm{K}}}{(M-1)(K-1)-1}\right)
$$

Proof: The proof sketch appears in Appendix E.

Theorem 9 (Performance Benchmark for 2 Selected Users): For a system with an $M$-antenna BS and $K$ singleantenna users having large SINR targets, a lower bound on the average minimum transmit power, in case of $K_{s}=2$ active users, required to achieve SINR targets is given by:

$$
p_{\mathrm{L}}(2, K)=\gamma\left(K \alpha_{\mathrm{M}, K-1}-(K-1)\left(1-\frac{M-1}{(M-1)(K-1)-1}\right) \alpha_{\mathrm{M}, \mathrm{K}}\right)
$$

Proof: The proof is outlined in Appendix F.

It is worth noting that this lower bound is for transmit power averaged over channel realizations, as there could be certain realizations that may require lesser power to meet users' SINR targets.

\section{Performance comparison}

In this section, we compare the performance of user selection algorithms treated in previous sections when the metric of interest is the average minimum transmit power required to satisfy users' SINR constraints. For analysis and comparison, separate curves have been plotted for all the user selection algorithms for two cases:

(i) upper bounds of the power using the analytical results derived in previous section and

(ii) Monte Carlo simulation curves representing the true average minimum power.

The curves show that upper bound results are reasonably close to the true powers.

For all the simulation setups, optimal user selection and its average minimum power have been determined through exhaustive search over all possible user combinations. These curves are represented as "OPT" in the following figures. Furthermore, for the case of two selected users, the lower bound of the average minimum power has been plotted using Theorem 9 .

For all the scenarios studied, SUS simulation curves fully overlap the OPT curves highlighting the fact that SUS is indeed the greedy selection algorithm for power 
minimization objective, as indicated in the discussion following Lemma 1.

\section{A. The case of $K_{s}=2$ selected users}

The plot of average minimum transmit power required to attain specific SINR targets $\gamma$ versus the number of antennas at the BS appears in Figure 1 for the considered user selection algorithms. A minor gap is visible between the SUS simulation curve (true SUS power) and the SUS analytical upper bound as the orthogonalized norm distributions were bounded in the proof (see Appendix D for details). We remark that SUS performs better than other user selection schemes, but with the increase in the number of transmit antennas, NUS also performs very well. The similar behavior was observed in [20], and the reason comes from the fact that with the increase in the number of transmit antennas, users' channels start becoming (close to) spatially orthogonal (this is clearly visible through the angle distributions such as $F_{\sin ^{2} \theta_{i}}(M ; x)$ in Appendix B). Furthermore increase in $M$ causes to increase the dimensions of the transmit signal space that provides the BS more flexibility to choose appropriate BF vectors for any fixed $K_{s}$.

Figure 2 plots the curves of the average minimum transmit power versus the number of users for a fixed number of transmit antennas. SUS simulation curve overlaps OPT exhaustive search curve, but we remark that NUS does not behave very well in this scenario, because it chooses users without paying any attention to their spatial orthogonality, which may affect significantly the interference observed by the selected users. The degradation incurred by NUS w.r.t. OPT increases further with large $K$.

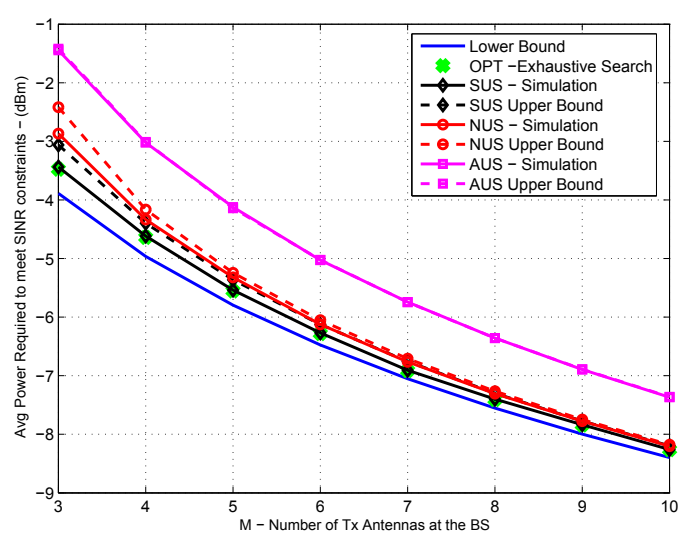

Figure 1 Avg. Min. Transmit Power vs. $M$ for $K=10, K_{S}=2, \gamma=$ $10 \mathrm{~dB}, \sigma^{2}=\mathbf{0 . 1}$. The curves show that SUS fully overlaps the OPT curve. NUS also performs well and moves close to OPT curve with increasing number of transmit antennas.

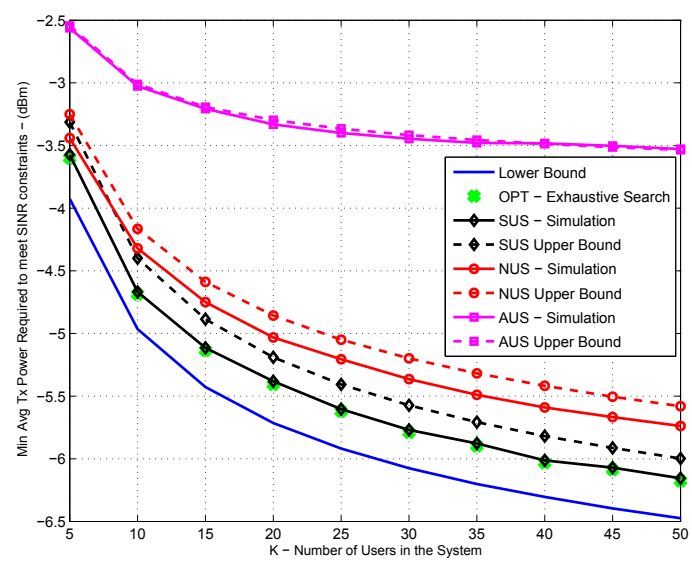

Figure 2 Avg. Min. Transmit Power vs. Nb. of Users for $M=4$, $K_{S}=2, \gamma=10 \mathrm{~dB}, \sigma^{2}=\mathbf{0 . 1}$. The curves show that SUS completely overlaps the optimal curve. NUS shows performance degradation as the number of users increases.

\section{B. The case of $K_{s}=4$ selected users}

We plot the average minimum transmit power required to achieve certain SINR targets versus the number of transmit antennas and versus the number of system users in Figures 3 and 4, respectively, for the user selection algorithms of interest. For both of these plots, the number of selected users is 4 . We observe the same behavior as observed for the case of 2 selected users. SUS gives the same performance as of the exhaustive search approach. For large number of transmit antennas, NUS performs close to the optimal, even AUS achieves a reasonable performance.

On the other hand, for a fixed number of transmit antennas at the BS, when the number of users present in the system increases, NUS shows an increasing performance degradation. The reason is that NUS captures

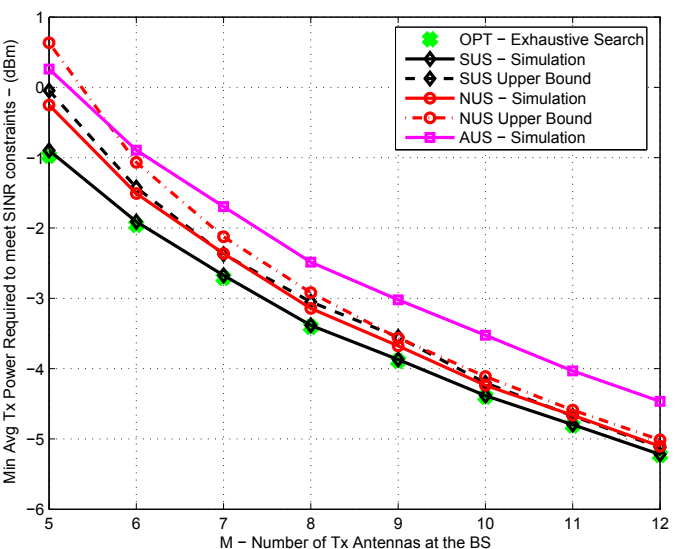

Figure 3 Avg. Min. Transmit Power vs. $M$ for $K=10, K_{S}=4, \gamma=$ $10 \mathrm{~dB}, \sigma^{2}=\mathbf{0 . 1}$. The curves show that SUS completely overlaps the exhaustive search result. Performance gap between NUS and OPT decreases for large number of transmit antennas. 


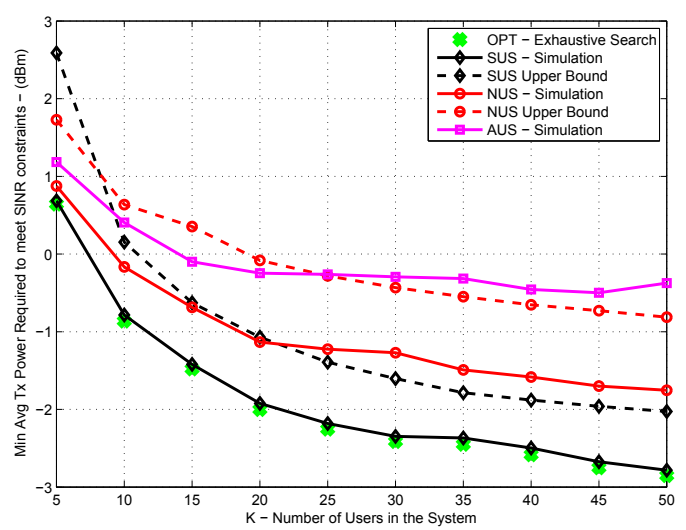

Figure 4 Avg. Min. Transmit Power vs. Nb. of Users for $M=5$, $K_{S}=4, \gamma=10 \mathrm{~dB}, \sigma^{2}=\mathbf{0 . 1}$. SUS Simulation curve overlaps the OPT exhaustive curve but NUS-Simulation shows more performance degradation with increasing number of users.

the raw aspect of multi-user diversity, which governs only the self-signal power, but pays no attention to the interuser spatial separation, which might have a larger impact on the interference power. The worst performance of AUS is expected, as it pays no attention to the strength of the selected users, which is quite important for power minimization objective. Again, Figure 4 shows SUS giving the performance of exhaustive OPT curve when all other selection schemes are getting worse.

\section{Power minimization and power efficiency}

To have an idea about how much power BS is spending per user and how does this power per user scale with the number of simultaneously scheduled users $K_{s}$, we plot the average minimum power per user versus the number of users $K_{s}$ in Figure 5. To get the power per user averaged in each scheduling group, the average minimum power per user values have been obtained by dividing the total minimum BS power to satisfy SINR targets of $K_{s}$ users by $K_{s}$. As expected, even for this power per user metric, SUS shows much better performance than NUS or AUS. AUS shows very bad performance and is highly power inefficient even for small number of simultaneously scheduled users. We remark a large increase in per-user power for a fixed SINR target with increase in number of simultaneously scheduled users. This shows that communication is power efficient when $K_{s}$ is much smaller than $M$ and becomes quite power inefficient when the number of scheduled users is close to the number of BS antennas.

\section{Conclusions}

In this paper, we have studied the performance of various user selection algorithms in terms of the average minimum transmit power required to satisfy specific SINR targets at users' side. General closed-form expressions of

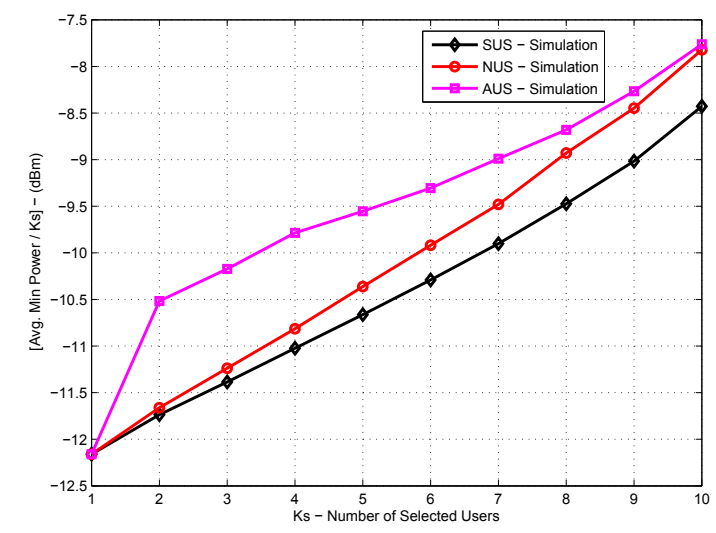

Figure 5 Avg. Min. Transmit Power per User vs. Nb. of Selected Users for $M=10, K=20, \gamma=10 \mathrm{~dB}, \sigma^{2}=0.1$. The curves show that communication becomes highly power inefficient when the number of simultaneously scheduled users $K_{s}$ grows close to the number of BS antennas $M$.

the average minimum transmit power for the three user selection algorithms, namely SUS, NUS, and RUS, were derived when any number of users are selected for simultaneous transmission. Furthermore, for the special case when only two users are selected for simultaneous transmission, similar expressions are derived for AUS and a power lower bound, which serves to benchmark other selection algorithms. SUS, which has been shown to behave close to optimal for the sum rate maximization objective under fixed power constraint, shows equally attractive performance in this dual problem setting of transmit power minimization to achieve hard SINR targets. An interesting observation is the fact that SUS is the greedy algorithm for power minimization objective. For a fixed number of users and increasing number of transmit antennas, NUS performs close to SUS. In the complementary setting of fixed number of BS transmit antennas, the performance of NUS degrades with an increase in the number of system users. Furthermore, power efficiency curves provided in this study may help the network operators decide how many users should be scheduled for simultaneous transmission per resource block.

\section{Appendix A}

Proof of Lemma 1: minimum instantaneous transmit power

The instantaneous transmit power required to achieve the SINR targets at $K_{s}$ active users

having channels $\mathbf{h}_{1}, \mathbf{h}_{2}, \ldots, \mathbf{h}_{\mathbf{K}_{\mathrm{s}}}$ is given by the following expression from [15]

$$
p_{\mathrm{tx}}\left(\mathbf{h}_{\mathbf{1}}, \mathbf{h}_{2}, \ldots, \mathbf{h}_{\mathbf{K}_{\mathrm{s}}}\right)=\sum_{i=1}^{K_{s}} \frac{\gamma_{i}}{\mathbf{h}_{\mathbf{i}}^{\dagger} \mathbf{Z}_{\mathbf{i}}^{-1} \mathbf{h}_{\mathbf{i}}},
$$


where $\mathbf{Z}_{\mathbf{i}}$ is given by the following expression

$$
\mathbf{Z}_{\mathbf{i}}=\mathbf{I}_{\mathbf{M}}+\sum_{j=1}^{i-1} p_{j} \mathbf{h}_{\mathbf{j}} \mathbf{h}_{\mathbf{j}}^{\dagger}
$$

The minimum power allocated to the stream of 1 st user to achieve its SINR target $\gamma_{1}$ is

$$
p_{1}=\frac{\gamma_{1}}{\mathbf{h}_{1}^{\dagger} \mathbf{I}_{\mathbf{M}} \mathbf{h}_{1}}=\frac{\gamma_{1}}{\left\|\mathbf{h}_{\mathbf{1}}\right\|^{2}} \text {. }
$$

The power allocated to the stream of 2 nd user to achieve its SINR target $\gamma_{2}$ is

$$
p_{2}=\frac{\gamma_{2}}{\mathbf{h}_{2}^{\dagger} \mathbf{Z}_{2}^{-1} \mathbf{h}_{2}}
$$

Non-identity $\mathbf{Z}_{2}^{-1}$ appears because user 2 will see the interference from the stream of 1 st user.

$$
\mathbf{Z}_{2}^{-1}=\left(\mathbf{I}_{\mathbf{M}}+p_{1} \mathbf{h}_{1} \mathbf{h}_{1}^{\dagger}\right)^{-1}
$$

Applying the matrix inversion lemma (MIL) to the right-hand side (R.H.S.) of the above equation, we get

$$
\begin{aligned}
\mathbf{Z}_{2}^{-1} & =\mathbf{I}_{\mathbf{M}}-p_{1} \mathbf{h}_{1}\left(1+p_{1}\left\|\mathbf{h}_{1}\right\|^{2}\right)^{-1} \mathbf{h}_{1}^{\dagger} \\
& \geq \mathbf{I}_{\mathbf{M}}-p_{1} \mathbf{h}_{\mathbf{1}}\left(p_{1}\left\|\mathbf{h}_{\mathbf{1}}\right\|^{2}\right)^{-1} \mathbf{h}_{\mathbf{1}}^{\dagger} .
\end{aligned}
$$

For two positive definite matrices $\mathbf{A}$ and $\mathbf{B}, \mathbf{A}>\mathbf{B}$ implies that A - B is positive definite [34]. As $p_{1}\left\|\mathbf{h}_{\mathbf{1}}\right\|^{2}$ $=\gamma_{1}$ from Equation (19), this inequality will become tight when the SINR target $\gamma_{1}$ is large. $\mathbf{Z}_{2}^{-1}$ becomes

$$
\mathbf{Z}_{2}^{-1} \geq \mathbf{I}_{M}-p_{1} \mathbf{h}_{1}\left(p_{1}|| \mathbf{h}_{1} \|^{2}\right)^{-1} \mathbf{h}_{1}^{\dagger}=\mathbf{I}_{M}-\frac{\mathbf{h}_{1} \mathbf{h}_{1}^{\dagger}}{\left\|\mathbf{h}_{1}\right\|^{2}}
$$

This renders

$$
\begin{aligned}
\mathbf{h}_{2}^{\dagger} \mathbf{Z}_{2}^{-1} \mathbf{h}_{2} & \geq\left\|\mathbf{h}_{2}\right\|^{2}-\frac{\left|\mathbf{h}_{2}^{\dagger} \mathbf{h}_{1}\right|^{2}}{\|\left.\mathbf{h}_{1}\right|^{2}} \\
& =\left\|\mathbf{h}_{2}\right\|^{2}\left(1-\cos ^{2} \theta_{1}\right)=\left\|\mathbf{h}_{2}\right\|^{2} \sin ^{2} \theta_{1},
\end{aligned}
$$

where $\theta_{1}$ denotes the angle that $\mathbf{h}_{2}$ subtends with the 1 -dimensional subspace spanned by $\mathbf{h}_{\mathbf{1}}$. Hence, the power allocation was done over the stream of 2nd user, so that it achieves its SINR target $\gamma_{2}$ that would be

$$
p_{2} \leq \frac{\gamma_{2}}{\left\|\mathbf{h}_{2}\right\|^{2} \sin ^{2} \theta_{1}}
$$

The power allocated to the stream of 3rd user is given by

$$
p_{3}=\frac{\gamma_{3}}{\mathbf{h}_{3}^{\dagger} \mathbf{Z}_{3}^{-1} \mathbf{h}_{3}}
$$

with

$$
\mathbf{Z}_{3}^{-1}=\left(\mathbf{I}_{\mathbf{M}}+p_{1} \mathbf{h}_{1} \mathbf{h}_{1}^{\dagger}+p_{2} \mathbf{h}_{2} \mathbf{h}_{2}^{\dagger}\right)^{-1} \text {. }
$$

Taking $\hat{\mathbf{h}}_{\mathbf{i}}=\sqrt{p_{i}} \mathbf{h}_{\mathbf{i}}$ and then $\mathbf{H}_{12}=\left[\hat{\mathbf{h}}_{\mathbf{1}} \hat{\mathbf{h}}_{\mathbf{2}}\right]$, we get $\mathbf{Z}_{3}=\left(\mathbf{I}_{M}+\mathbf{H}_{12} \mathbf{H}_{12}^{\dagger}\right)$. Applying MIL to the R.H.S. of the above equation, $\mathbf{Z}_{3}^{-1}$ can be written as

$$
\begin{aligned}
\mathbf{Z}_{3}^{-1} & =\mathrm{I}_{\mathrm{M}}-\mathrm{H}_{12}\left(\mathrm{I}_{2}+\mathrm{H}_{12}^{\dagger} \mathrm{H}_{12}\right)^{-1} \mathbf{H}_{12}^{\dagger} \\
& \geq \mathrm{I}_{\mathrm{M}}-\mathrm{H}_{12}\left(\mathrm{H}_{12}^{\dagger} \mathrm{H}_{12}\right)^{-1} \mathbf{H}_{12}^{\dagger}
\end{aligned}
$$

where it is trivial to show that $\left(\mathbf{H}_{12}^{\dagger} \mathbf{H}_{12}\right)^{-1}-\left(\mathbf{I}_{2}+\mathbf{H}_{12}^{\dagger} \mathbf{H}_{12}\right)^{-1}$ is positive semi-definite. $\mathbf{I}_{2}$ matrix adds 1 to the diagonal elements of $\mathbf{H}_{12}^{\dagger} \mathbf{H}_{12}$, which are $p_{1}|| \mathbf{h}_{\mathbf{1}} \|^{2}$, and $p_{2}|| \mathbf{h}_{\mathbf{2}} \|^{2}$, respectively. As $p_{1} \|$ $\mathbf{h}_{1} \|^{2}=\gamma_{1}$, and $p_{2}\left\|\mathbf{h}_{2}\right\|^{2}=\frac{\gamma_{2}}{\sin ^{2} \theta_{1}}>\gamma_{2}$, the above inequality will be tight for large SINR targets. As $\mathbf{H}_{12}\left(\mathbf{H}_{12}^{\dagger} \mathbf{H}_{12}\right)^{-1} \mathbf{H}_{12}^{\dagger}$ is the projection matrix over the column space of $\mathbf{H}_{\mathbf{1 2}}$, i.e., over the space spanned by $\mathbf{h}_{\mathbf{1}}$ and $\mathbf{h}_{2}$, the product $\mathbf{h}_{3}^{\dagger} \mathbf{Z}_{3}^{-1} \mathbf{h}_{\mathbf{3}}$ gives the energy of the channel $\mathbf{h}_{\mathbf{3}}$ projected over the subspace orthogonal to that spanned by $\mathbf{h}_{\mathbf{1}}$ and $\mathbf{h}_{\mathbf{2}}$.

$$
\begin{aligned}
\mathbf{h}_{3}^{\dagger} \mathbf{Z}_{3}^{-1} \mathbf{h}_{3} & \geq\left\|\mathbf{h}_{3}\right\|^{2}-\mathbf{h}_{3}^{\dagger} \mathbf{H}_{12}\left(\mathbf{H}_{12}^{\dagger} \mathbf{H}_{12}\right)^{-1} \mathbf{H}_{12}^{\dagger} \mathbf{h}_{3} \\
& =\left\|\mathbf{h}_{3}\right\|^{2}\left(1-\cos ^{2} \theta_{2}\right)=\left\|\mathbf{h}_{3}\right\|^{2} \sin ^{2} \theta_{2}
\end{aligned}
$$

where $\theta_{2}$ is the angle subtended by $\mathbf{h}_{3}$ with the 2 -dimensional subspace spanned by $\mathbf{h}_{\mathbf{1}}$ and $\mathbf{h}_{\mathbf{2}}$. Hence, the power allocated to the stream of 3rd user to raise its SINR to $\gamma_{3}$ is given by

$$
p_{3} \leq \frac{\gamma_{3}}{\left\|\mathbf{h}_{3}\right\|^{2} \sin ^{2} \theta_{2}} .
$$

In fact, this procedure generalizes to any number of users, and the power allocated to the stream of $i$ th active user is given by

$$
p_{i} \leq \frac{\gamma_{i}}{\left\|\mathbf{h}_{\mathbf{i}}\right\|^{2} \sin ^{2} \theta_{(i-1)}},
$$

where $\theta_{(i-1)}$ is the angle that $\mathbf{h}_{\mathbf{i}}$ makes with the $(i$ 1)-dimensional subspace spanned by $\mathbf{h}_{\mathbf{1}}, \mathbf{h}_{\mathbf{2}}, \ldots, \mathbf{h}_{\mathbf{i}-\mathbf{1}}$ as a function of encoding order. Summing the powers allocated to all active users, the minimum transmit power to achieve SINR targets at $K_{s}$ users is given by:

$$
p_{\mathrm{tx}}\left(\mathbf{h}_{1}, \mathbf{h}_{2}, \ldots, \mathbf{h}_{\mathrm{K}_{\mathrm{s}}}\right) \leq \sum_{i=1}^{K_{s}} \frac{\gamma_{i}}{\left\|\mathbf{h}_{\mathbf{i}}\right\|^{2} \sin ^{2} \theta_{(i-1)}} .
$$




\section{Appendix B}

\section{Some useful distributions}

In this appendix, we give some useful cumulative distribution functions (CDF), for which probability density functions (PDF) can be computed by simple differentiation.

\section{A. Channel norm distributions}

Most of the channel norm (squared) distributions given in this subsection are known relations, others have been computed using the tools from order statistics [35]. If all the users have $M$ - dimensional spatially i.i.d. complex Gaussian channels, the squared channel norm, || $\mathbf{h}_{\mathbf{i}} \|^{2}$ for any $i$, is $\chi^{2}$ distributed with $2 M$ degrees of freedom. This CDF can be represented as [33]

$$
F_{\| \mathbf{h}||^{2}}(M ; x)=G(M, x),
$$

where $\mathrm{G}$ denotes the regularized Gamma function [33], and is defined as

$$
G(M, x)=\frac{1}{\Gamma(M)} \int_{0}^{x} e^{-t} t^{M-1} d t
$$

The PDF corresponding to $\operatorname{CDF} F_{\| \mathbf{h}||^{2}}(M ; x)$ is given by

$$
f_{|| \mathbf{h}||^{2}}(M ; x)=\frac{e^{-x} x^{M-1}}{\Gamma(M)} .
$$

We also need the CDF for $r$ th largest squared channel norm among $\mathrm{K}$ independent channel vectors. The CDF of the $r$ th largest order statistic among $K$ i.i.d. variables, each of which has the CDF of $F_{\|\mathbf{h}\|^{2}}(M ; x)$, is given by [35]

$$
F_{\|\mid \mathbf{h}\|^{2}}(M, r, K ; x)=\sum_{j=K+1-r}^{K}\left(\begin{array}{c}
K \\
j
\end{array}\right)\left[F_{\|\mid \mathbf{h}\|^{2}}(M ; x)\right]^{j}\left[1-F_{\|\left.\mathbf{h}\right|^{2}}(M ; x)\right]^{K-j}
$$

The CDFs for the largest, the second largest, thirdorder, or fourth-order statistics can be determined easily by plugging in appropriate value of $r$. The distribution of any random user among $K$ users that does not have the largest norm can be specified as (from [20])

$$
F_{|| \mathbf{h} \|^{2}}(M, 1, K ; x)=\frac{K}{K-1} F_{|| \mathbf{h} \|^{2}}(M ; x)-\frac{1}{K-1}\left[F_{|| \mathbf{h}||^{2}}(M ; x)\right]^{K}
$$

where 1 stands for a random user which is not the first order statistic.

\section{B. Channel direction distributions}

In this subsection, we give some useful distributions of the $\sin ^{2}$ and $\cos ^{2}$ of the angle between a vector and a subspace. If we have $K$ i.i.d. $M$-dimensional Gaussian distributed vectors, i.e., $\mathbf{h}_{\mathbf{i}} \in \mathbb{C}^{M}$ for user $i$, we can compute the distribution of the $\sin ^{2}$ and $\cos ^{2}$ of the angle between one vector and the subspace spanned by a subset of the other vectors. This angle is defined as the (sole) principal angle of vector $\mathbf{h}_{\mathbf{i}}$ with the subspace
[36]. For a channel vector $\mathbf{h}_{\mathbf{j}}$ and a subspace spanned by $i$ independent Gaussian vectors $\mathbf{h}_{1}, \mathbf{h}_{2}, \ldots, \mathbf{h}_{\mathbf{i}}$, if $\theta_{i}$ denotes the angle $\mathbf{h}_{\mathbf{j}}$ subtends with this $i$-dimensional subspace, the projection of $\mathbf{h}_{\mathbf{j}}$ on this subspace $\cos ^{2} \theta_{i}$ has a $\beta$ distribution with parameters $i$ and $M-i$ (see $[37,38]$ for details). $\sin ^{2} \theta_{i}=1-\cos ^{2} \theta_{i}$ also has the beta distributions with shift of parameters $\beta(M-i, i)$. The CDF of $\sin ^{2} \theta_{i}$, denoted as $F_{\sin ^{2} \theta_{i}}(M ; x)$ is given by

$$
F_{\sin ^{2} \theta_{i}}(M ; x)=\frac{B_{x}(M-i, i)}{B(M-i, i)}=\frac{(M-1) !}{(M-i-1) !(i-1) !} \int_{t=0}^{x} t^{M-i-1}(1-t)^{i-1} d t,
$$

where $B$ and $B_{x}$ denote the beta function and the regularized beta function, respectively $[33,38]$. If $\theta_{1}$ denotes the angle that an $M$-dimensional vector $\mathbf{h}_{\mathbf{j}}$ makes with an independent vector $\mathbf{h}_{\mathbf{1}}$ (one-dimensional subspace), the distribution of $\sin ^{2} \theta_{1}$ is given by

$$
F_{\sin ^{2} \theta_{1}}(M ; x)=x^{M-1} .
$$

The CDFs of projection with larger subspaces can be obtained by putting the appropriate value for the dimension of the subspace in Equation (38), w.r.t. which orthogonalization is performed.

We saw that the energy in the orthogonal projection of one vector over another independent vector assumes the CDF of $F_{\sin ^{2} \theta_{1}}(M ; x)$. If there are $K$ such projections (each having the CDF of $F_{\sin ^{2} \theta_{1}}(M ; x)$ ), the CDF of the largest (1st order) projection is given by

$$
F_{\sin ^{2} \theta_{1}}(M, 1, K ; x)=\left[F_{\sin ^{2} \theta_{1}}(M ; x)\right]^{K}=x^{K(M-1)} .
$$

\section{Appendix C}

\section{Proof of Theorem 1: norm-based user selection}

In the proof of the theorem for NUS and the rest of the appendices, we make extensive use of the useful CDFs that have been grouped together in Appendix B, so we highly encourage the readers to go through the previous appendix for proper understanding of these proofs and the notation associated to those CDFs.

For NUS, the users are selected as described in Section 4 . The squared norm of the $i$ th selected user $\pi^{N}(i)$ is the $i$ th largest among $K$ users, and hence is distributed as $F_{|| \mathbf{h}||^{2}}(M, i, K ; x)$. We reproduce the expression for minimum transmit power below

$$
p_{\mathrm{tx}}\left(\mathbf{h}_{\mathbf{1}}, \mathbf{h}_{2}, \ldots, \mathbf{h}_{\mathbf{K}_{\mathrm{s}}}\right) \leq \gamma \sum_{i=1}^{K_{s}} \frac{1}{\left\|\mathbf{h}_{\mathbf{i}}\right\|^{2} \sin ^{2} \theta_{(i-1)}}
$$

As NUS is solely based upon users' channel norms and the Gaussian distributed vectors have independent norms and directions, the directional properties of these vectors are as if they are randomly selected. Hence, $\sin ^{2}$ $\theta_{i}$, where $\theta_{i}$ is the angle a vector makes with an 
independent $i$-dimensional subspace, is distributed as $F_{\sin ^{2} \theta_{i}}(M ; x)$ as detailed in Appendix B.

For the above expression, encoding order is $K_{s}$ to 1 . In terms of NUS indices, this encoding order is from $\pi^{N}(1)$ to $\pi^{N}\left(K_{s}\right)$, i.e., the strongest user $\pi^{N}(1)$ will face the interference of all other users who get encoded later. For the user whose signal gets decoded with $(i-1)$ interference streams would be the one encoded at $\left(K_{s}-i+1\right)$-th order. Hence, its NUS index would be $\pi^{N}\left(K_{s}-i+1\right)$, and its squared norm would be distributed as $F_{\|\mathbf{h}\|^{2}}\left(M, K_{s}+1-i, K ; x\right)$. As its interference subspace is $(i-1)$-dimensional, the $\sin ^{2}$ of its angle with this subspace is distributed as $F_{\sin ^{2} \theta_{(i-1)}}(M ; x)$. If we denote the average transmit power, required to satisfy target SINRs at $K_{s}$ users selected through NUS from a pool of $K$ users, by $p_{\mathrm{N}}\left(K_{s}, K\right)$, this can be obtained by computing the expectations in the following expression:

$$
p_{\mathrm{N}}\left(K_{s}, K\right) \leq \gamma \sum_{i=1}^{K_{s}}\left(\mathbb{E}_{F_{\|\left.||\right|^{2}}\left(M, K_{s}+1-i, K ; x\right)}\left[\frac{1}{x}\right] \mathbb{E}_{F_{\sin ^{2} \theta_{(i-1)}}(M ; x)}\left[\frac{1}{x}\right]\right) .
$$

Like Lemma 1, this bound will be tight for large SINR targets.

\section{A. NUS for 2 users}

When only two users are selected through NUS for simultaneous transmission, the ordering strategy remains the same (the weaker user gets decoded with no interference), and the average power required can be computed by taking only the first two terms of the general NUS transmit power expression.

$$
p_{\mathrm{N}}(2, K) \leq \gamma\left(\mathbb{E}_{F_{\left.|| h\right|^{2}(M, 2, K ; x)}}\left[\frac{1}{x}\right]+\mathbb{E}_{F_{\left.|| h\right|^{2}(M, 1, K ; x)}}\left[\frac{1}{x}\right] \mathbb{E}_{F_{\sin ^{2} \theta_{1}(M ; x)}}\left[\frac{1}{x}\right]\right)
$$

The PDF corresponding to $\operatorname{CDF} F_{|| \mathbf{h}||_{2}}(M, 1, K ; x)$ (obtained by its differentiation) is given by

$$
f_{\| \mathbf{h}||^{2}}(M, 1, K ; x)=K[G(M, x)]^{K-1} \frac{e^{-x} x^{M-1}}{\Gamma(M)} .
$$

It allows us to compute the following expectation:

$$
\begin{aligned}
\mathbb{E}_{F_{|| \mathbf{|}||^{2}}(M, 1, K ; x)}\left[\frac{1}{x}\right] & =\int_{0}^{\infty}\left[\frac{1}{x}\right] K[G(M, x)]^{K-1} \frac{e^{-x} x^{M-1}}{\Gamma(M)} d x \\
& =\int_{0}^{\infty} K \frac{e^{-x} x^{M-2}}{\Gamma(M)}[G(M, x)]^{K-1} d x \\
& =\alpha_{\mathrm{M}, \mathrm{K}}
\end{aligned}
$$

where the last equality is the definition of the constant term $\alpha_{\mathrm{M}, \mathrm{K}}$, defined in Equation (11), which only depends upon the specific values of $M$ and $K$. Similarly, it can be shown that

$$
\mathbb{E}_{F_{\|\mathbf{h}\|^{2}(M, 2, K ; x)}}\left[\frac{1}{x}\right]=K \alpha_{\mathrm{M}, \mathrm{K}-1}-(K-1) \alpha_{\mathrm{M}, \mathrm{K}} .
$$

The expectation concerning the angle distribution can also be computed as follows:

$$
\mathbb{E}_{F_{\sin ^{2} \theta_{1}(M ; x)}}\left[\frac{1}{x}\right]=\int_{0}^{1}\left[\frac{1}{x}\right](M-1) x^{M-2} d x=\frac{M-1}{M-2} .
$$

Combining the results of these expectations in Equation (43) and doing some rearrangements gives the result of Corollary 2. The average transmit power when 4 users are selected through NUS (Corollary 3) can be computed, similarly, by taking the first four terms from the general NUS average transmit power expression.

\section{Appendix D}

Proof of Theorem 4: semi-orthogonal user selection

For computing the average power required to satisfy SINR targets, we need the CDFs of the random variables as a consequence of the use of SUS algorithm in the expression given below:

$$
p_{\mathrm{tx}}\left(\mathbf{h}_{1}, \mathbf{h}_{2}, \ldots, \mathbf{h}_{\mathbf{K}_{\mathrm{s}}}\right) \leq \gamma \sum_{i=1}^{K_{\mathrm{s}}} \frac{1}{\left\|\mathbf{h}_{\mathbf{i}}\right\|^{2} \sin ^{2} \theta_{(i-1)}}
$$

In SUS, the user selected at ith iteration $\pi^{S}(i)$ is the one with the largest channel norm on the null space of the subspace spanned by the channel vectors of $i-1$ earlier selected users. The $K_{s}$ selected users through SUS need to be DPC encoded. The encoding order in the above expression is $K_{s}$ to 1 . The optimal encoding order in terms of SUS indices would be $\pi^{S}(1)$ to $\pi^{S}$ $\left(K_{s}\right)$, so that the weaker user gets the least interference [25]. Unfortunately, it is not possible to determine the resulting CDFs with this encoding order, so we use the suboptimal encoding order from $\pi^{S}\left(K_{s}\right)$ to $\pi^{S}(1)$, where $\pi^{S}(i)$ will receive the interference of $i-1$ users, indexed from $\pi^{S}(i-1)$ to $\pi^{S}(1)$. This encoding order aligns the SUS metric, orthogonalized squared norm || $\mathbf{h}_{\mathbf{i}}||^{2} \sin ^{2} \theta_{(i-1)}$, with the denominator of above expression. The average power computation requires the CDF of $\left\|\mathbf{h}_{\mathbf{i}}\right\|^{2} \sin ^{2} \theta_{(i-1)}$. Statistically, this represents the largest norm among $K$ - ( $i-1)$ norms in $M-(i-1)$ dimensional subspace, which is the null space of the subspace spanned by the channel vectors of $i-1$ earlier selected users in previous SUS iterations. Unfortunately, this CDF is hard to compute, so we ease the computation using [[39], Lemma 3], which was also used in [[11], Appendix III]. Following [39], we orthogonalize all the channel vectors w.r.t. $i-1$ arbitrary vectors, so for each of them, the squared norm is $\chi^{2}$ distributed with $2(M-i+1)$ degrees of freedom, denoted as $F_{|| \mathbf{h}||^{2}}(M-i+1 ; x)$. Let us denote the projection of $\mathbf{h}_{\mathbf{j}}$ on the null space of those $i-1$ arbitrary 
vectors by $\tilde{\mathbf{h}}_{\mathbf{j}}$, then $i$ th largest norm of these orthogonalized vectors will be

$$
\left\|\widehat{\mathbf{h}}_{\mathbf{i}}\right\|^{2}=i \text { th } \max \left\|\widetilde{\mathbf{h}}_{\mathbf{j}}\right\|^{2}, \quad j=1, \ldots, K .
$$

The distribution of $\left\|\hat{\mathbf{h}}_{\mathbf{i}}\right\|^{2}$ is given by $F_{\|\mathbf{h}\|^{2}}(M-i+1, i, K ; x)$, the $i$ th largest of $K$ instances in $(M-i+1)$-dimensional space. Lemma 3 in [39] shows that statistically $\left\|\hat{\mathbf{h}}_{\mathbf{i}}\right\|^{2}$ is smaller than $\left\|\mathbf{h}_{\mathbf{i}}\right\|^{2} \sin ^{2} \theta_{(i-1)}$. Thus, the average power required to satisfy target SINRs at $K_{s}$ users selected from a pool of $K$ users through SUS is given by:

$$
\begin{aligned}
p_{\mathrm{S}}\left(K_{s}, K\right) & \leq \gamma \sum_{i=1}^{K_{s}}\left(\mathbb{E} \frac{1}{\left\|\mathbf{h}_{\mathbf{i}}\right\|^{2} \sin ^{2} \theta_{(i-1)}}\right) \\
& \leq \gamma \sum_{i=1}^{K_{s}}\left(\mathbb{E} \frac{1}{\left\|\widehat{\mathbf{h}}_{\mathbf{i}}\right\|^{2}}\right)
\end{aligned}
$$

The second inequality is the consequence of lower bounding the orthogonalized squared norms by the use of [[39], Lemma 3]. As CDF for $\left\|\hat{\mathbf{h}}_{\mathbf{i}}\right\|^{2}$ is known, we get the final result

$$
p_{\mathrm{S}}\left(K_{s}, K\right) \leq \gamma \sum_{i=1}^{K_{S}}\left(\mathbb{E}_{F_{\|\left.\mathbf{h}\right|^{2}}(M-i+1, i, K ; x)}\left[\frac{1}{x}\right]\right) .
$$

The average transmit power expressions when 2 or 4 users are selected through SUS (Corol-laries 5 and 6) can be obtained by taking the first two or four terms, respectively, from the general SUS average power expression and computing the integrals.

\section{Appendix E}

\section{Proof of Theorem 8: angle-based user selection}

When 2 users are selected through AUS, the first selected user $\pi^{A}(1)$ is the strongest user among $K$ users, whose squared norm is distributed as $F_{|| \mathbf{h}|| 2}(M, 1, K ; x)$. As norms and directions are independent, the distribution of $\sin ^{2}$ of the angle that other $K-1$ vectors individually make with the first selected vector (1-dimensional subspace) all follow the distribution of $F_{\sin ^{2} \theta_{1}}(M ; x)$. The second selected user $\pi^{A}(2)$ among $K-1$ users is the one making the largest angle with the first user. Hence, statistically, $\sin ^{2}$ of this angle is the largest order statistic among $K-1$ instances and is distributed as $F_{\sin ^{2} \theta_{1}}(M, 1, K-1 ; x)$ (see Appendix B for details). The squared norm of $\pi^{A}(2)$ is distributed as the squared norm of any random user which is not the user with the largest norm, and hence the CDF is $F_{\left.|| \mathbf{h}\right|^{2}}(M, 1, K ; x)$, (see Equation (37) in Appendix B). We keep the same user ordering as detailed in NUS proof such that weaker user's signal gets decoded with lesser interference. The average transmit power for this user selection is given by:

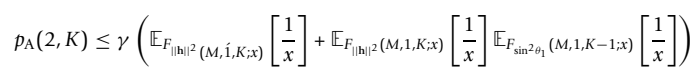

This will give the result for the case of two users. Unfortunately, we could not extend the average power required with AUS to the general case of $K_{s}$ users due to added complexity.

\section{Appendix $\mathbf{F}$}

\section{Proof of Theorem 9: performance benchmark}

To compute a lower bound on the average minimum transmit power required to satisfy SINR targets of $\gamma$, we assume that SINR targets are large and the inequality in Lemma 1 is tight. The two selected users are assumed to have the two largest norms, as in NUS having the CDFs of $F_{\|\mathbf{h}\| \mid}(M, 1, K ; x)$ and $F_{\|\mathbf{h}\|^{2}}(M, 2, K ; x)$. Further, the angle between their channel vectors is assumed to be the largest possible angle as in AUS, distributed as $F_{\sin ^{2} \theta_{1}}(M, 1, K-1 ; x)$. The similar strategy was employed in [[21], Lemma 2] to obtain a bound in the non-DPC case. Hence, with optimal ordering (the weaker user gets decoded with no interference), the lower bound on the average transmit power can be obtained by computing the expectations in the following expression:

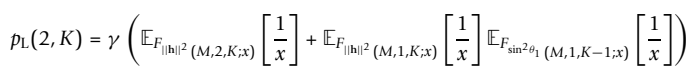

\section{Acknowledgements}

The research work carried out at Intel Mobile Communications leading to these results has received funding from the European Community's Seventh Framework Program (FP7/2007-2013) SACRA project (grant agreement 249060).

EURECOM's research is partially supported by its industrial members: BMW Group, Swiss-com, Cisco, ORANGE, SFR, ST Ericsson, Thales, Symantec, SAP, Monaco Telecom. The research of EURECOM is also supported in part by the EU FP7 projects CROWN, SACRA, and WHERE2.

\section{Author details}

${ }^{1}$ Intel Mobile Communications, France ${ }^{2}$ Mobile Communications Department of EURECOM, France

\section{Competing interests}

The authors declare that they have no competing interests.

Received: 1 December 2010 Accepted: 11 October 2011

Published: 11 October 2011

\section{References}

1. T Cover, Broadcast channels. IEEE Trans Inf Theory 18, 2-14 (1972). doi:10.1109/TIT.1972.1054727

2. IE Telatar, Capacity of multi-antenna Gaussian channels. Eur Trans Telecommun. 10, 585-595 (November 1999). doi:10.1002/ett.4460100604

3. GJ Foschini, MJ Gans, On limits of wireless communications in a fading environment when using multiple antennas. Wirel Pers Commun. 6, 311-335 (1998). doi:10.1023/A:1008889222784

4. H Weingarten, Y Steinberg, S Shamai, The capacity region of the Gaussian multiple-input multiple-output broadcast channel. IEEE Trans Inf Theory 52, 3936-3964 (2006) 
5. P Viswanath, D Tse, Sum capacity of the multiple antenna Gaussian broadcast channel and uplink-downlink duality. IEEE Trans Inf Theory 49, 1912-1921 (2003). doi:10.1109/TIT.2003.814483

6. G Caire, S Shamai (Shitz), On the achievable throughput of a multiantenna Gaussian broadcast channel. IEEE Trans Inf Theory 49, 1691-1706 (2003). doi:10.1109/TIT.2003.813523

7. W Yu, JM Cioffi, Sum capacity of Gaussian vector broadcast channels. IEEE Trans Inf Theory 50, 1875-1892 (2004). doi:10.1109/TIT.2004.833336

8. M Costa, Writing on dirty paper. IEEE Trans Inf Theory 29, 439-441 (1983). doi:10.1109/TIT.1983.1056659

9. Z Tu, R Blum, Multiuser diversity for a dirty paper approach. IEEE Commun Lett. 7, 370-372 (2003). doi:10.1109/LCOMM.2003.815652

10. G Dimic, N Sidiropoulos, On downlink beamforming with greedy user selection: performance analysis and a simple new algorithm. IEEE Trans Signal Process. 53, 3857-3868 (2005)

11. T Yoo, A Goldsmith, On the optimality of multiantenna broadcast scheduling using zero-forcing beamforming. IEEE J Sel Areas Commun. 24 528-541 (March 2006)

12. T Yoo, N Jindal, A Goldsmith, Multi-antenna downlink channels with limited feedback and user selection. IEEE J Sel Areas Commun. 25, 1478-1491 (September 2007)

13. F Rashid-Farrokhi, KJ Ray Liu, L Tassiulas, Transmit beamforming and power control for cellular wireless systems. IEEE J Sel Areas Commun. 16 1437-1450 (1998). doi:10.1109/49.730452

14. M Schubert, $\mathrm{H}$ Boche, Solution of the multi-user downlink beamforming problem with individual SINR constraints. IEEE Trans Veh Technol. 53, 18-28 (2004). doi:10.1109/TVT.2003.819629

15. M Schubert, Power-aware spatial multiplexing with unilateral antenna cooperation, PhD thesis (TU Berlin, 2003)

16. H Boche, M Schubert, A general duality theory for uplink and downlink beamforming, in Proceedings of IEEE Vehicular Technology Conference Fall (September 2002)

17. M Schubert, H Boche, Joint dirty paper pre-coding and downlink beamforming, in Proceedings of ISSSTA, 536-540 (September 2002)

18. E Karipidis, ND Sidiropoulos, Z-Q Luo, Quality of service and max-min fair transmit beamforming to multiple cochannel multicast groups. IEEE Trans Signal Process. 56, 1268-1279 (2008)

19. E Karipidis, ND Sidiropoulos, L Tassiulas, Joint QoS multicast power/ admission control and base station assignment: A geometric programming approach, in Proceedings of 5th IEEE Workshop on Sensor Array and MultiChannel Signal Processing (July 2008)

20. X Zhang, E Jorswieck, B Ottersten, A Paulraj, User selection schemes in multiple antenna broadcast channels with guaranteed performance, in Proceedings of IEEE Workshop on Signal Processing Advances in Wireless Communications (2007)

21. X Zhang, E Jorswieck, B Ottersten, A Paulraj, On the asymptotic optimality of opportunistic beamforming with hard SINR constraints. EURASIP J Adv Signal Process. 2009, 1-12. Special issue on Multiuser MIMO Transmission with Limited Feedback, Cooperation, and Coordination (2009)

22. U Salim, D Slock, Performance of different user selection algorithms for transmit power minimization, in Proceedings of Asilomar Conference on Signals, Systems and Computers (Pacific Grove, CA, USA, 2009)

23. R Zamir, S Shamai (Shitz), U Erez, Nested linear/lattice codes for structured multiterminal binning. IEEE Trans Inf Theory 48, 1250-1276 (2002). doi:10.1109/TIT.2002.1003821

24. U Erez, ST Brink, A close-to-capacity dirty paper coding scheme. IEEE Trans Inf Theory 51, 3417-3432 (2005). doi:10.1109/TIT.2005.855586

25. D Tse, P Viswanath, Fundamentals of Wireless Communications (Cambridge University Press, Cambridge, UK, 2005)

26. TM Cover, JA Thomas, Elements of Information Theory (Wiley, New York, 1991)

27. Z Shen, R Chen, JG Andrews, RW Heath, BL Evans, Low complexity user selection algorithms for multiuser mimo systems with block diagonalization. IEEE Trans Signal Process. 54, 3658-3663 (2006)

28. D Hammarwall, M Bengtsson, B Ottersten, Utilizing the spatial information provided by channel norm feedback in SDMA systems. IEEE Trans Signal Process. 56, 3278-3293 (2008)

29. S Han, C Yang, M Bengtsson, Al Perez-Neira, Channel norm-based user scheduler in coordinated multi-point systems, in Proceedings of IEEE Global Communications Conference (Honolulu, HI, USA, 2009)
30. R Knopp, PA Humblet, Information capacity and power control in single-cell multiuser communications, in Proceedings of International Conference on Communications, Seattle, USA, 331-335 (June 1995)

31. A Paulraj, in Diversity Techniques in the Mobile Communications Handbook, ed. by Gibson JD (CRC Press, 1999)

32. J Joung, AH Sayed, User selection methods for multiuser two-way relay communications using space division multiple access. IEEE Trans Wirel Commun. 9, 2130-2136 (2010)

33. M Abramowitz, A Stegun, Handbook of Mathematical Functions with Formulas, Graphs, and Mathematical Tables (Dover Publications, 1964)

34. RA Horn, CR Johnson, Matrix Analysis (Cambridge University Press, Cambridge, UK, 1985)

35. HA David, Order Statistics (Wiley, New York, 1980)

36. H Golub, CF Van Loan, Matrix Computations (John Hopkins Press, 1996)

37. N Jindal, Antenna combining for the MIMO downlink channel. IEEE Trans Wirel Commun. 10, 3834-3844 (2008)

38. AK Gupta, S Nadarajah, Handbook of Beta Distribution and Its Applications (CRC, 2004)

39. MA Maddah-Ali, MA Sadrabadi, AK Khandani, Broadcast in MIMO systems based on a generalized QR decomposition: Signaling and performance analysis. IEEE Trans Inf Theory 54, 1124-1138 (2008)

doi:10.1186/1687-1499-2011-127

Cite this article as: Salim and Slock: Average minimum transmit power to achieve SINR targets: performance comparison of various user selection algorithms. EURASIP Journal on Wireless Communications and Networking 2011 2011:127.

\section{Submit your manuscript to a SpringerOpen ${ }^{\circ}$ journal and benefit from:}

- Convenient online submission

- Rigorous peer review

- Immediate publication on acceptance

- Open access: articles freely available online

- High visibility within the field

- Retaining the copyright to your article

Submit your next manuscript at $\gg$ springeropen.com 